\title{
An Infographic Assignment to Translate Self-Care Therapeutics into Practical Application
}

Paul Malik, PharmD and Nardine Nakhla, PharmD

School of Pharmacy, University of Waterloo, Kitchener, Ontario, Canada

\section{ABSTRACT}

Innovation: An infographic assignment was developed and integrated into an advanced self-care therapeutics elective course in a School of Pharmacy to facilitate practical communication of dynamic and innovative approaches to patient care while supporting diversity in assessment.

Description: The 'Spotlight on Self-Care' assignment required pharmacy students to develop three infographic deliverables detailing comprehensive care for one minor ailment. The three deliverables were: 1) a magazine insert for healthcare professionals, 2) a patientfriendly handout, and 3) a pharmacists' companion practice tool. All deliverables were assessed by rubrics using consistent criteria, including: clinical content, logical presentation, research quality, visual elements and formatting. The five highest-scoring magazine inserts were offered publication in the Pharmacy Practice and Business Magazine after peer review.

Critical Appraisal: The submitted infographics put clinical content from the course into action by leveraging recent trends in effective communication. As an assessment, the infographic assignment recognized a unique profile of skills in the students that was statistically different from the profile of skills that was evaluated by the multiple-choice examinations. The key issues to address include reducing grading time requirements and developing strategies to detect copyrighted materials. Future investigations into the nature of the skills gained by the students through the exercise, as well as their perceptions regarding the professional value of the exercise, are important for refining the administration of this assignment.

Keywords: Infographic, Self-Care, Pharmacy Practice

\section{RATIONALE FOR THE INNOVATION}

Infographics (a clipped compound of "information" and "graphics") are visual representations of information, data or knowledge that are intended to communicate content quickly and clearly. Infographics appeal to the busy professional and are gaining popularity in business, government and healthcare for public advocacy. As an example, the Journal of the American Medical Association (JAMA) began publishing an infographic series in 2015 aimed to help healthcare professionals visualize trends and data relevant to health policy. ${ }^{1,2}$

Assignments to create infographics related to healthcare have been integrated into university courses in social work, nursing and public health..$^{3-5}$ These exercises have demonstrated utility for teaching students effective ways to present data. The final products often feature graphs, statistics and pie charts. In pharmacy practice, there is utility of infographics as an expansion to present approaches to patient care. In an infographic format, information becomes dynamic, engaging and quickly understood. The logical order is communicated clearly, visual aids enhance understanding at critical points and vibrant colors prioritize clinical information. Further translating these benefits into patient-friendly language will engage patients in pharmacies and clinics with communication materials of a kind often unavailable in healthcare.

Corresponding author: Nardine Nakhla, PharmD

Clinical Lecturer

School of Pharmacy, University of Waterloo

Kitchener, Ontario, Canada

Phone: (519) 888-4567 ext. 21340

Email: nnakhla@uwaterloo.ca
While infographic assignments leverage trends in modern and effective communication, they also offer an opportunity to support diversity in assessment. It is the responsibility of the educator to develop assessments that are directed toward developing the skills that are needed in practice. An infographic assignment that is centered on communicating approaches to patient care may assess valuable professional skills in students that are not otherwise evaluated by traditional computerbased examinations. Well-rounded assessment in higher education drives the development of well-rounded professionals. $^{6}$

\section{STATEMENT OF THE INNOVATION}

An infographic assignment was developed and integrated into an advanced self-care therapeutics elective course for third year students to facilitate practical communication of dynamic and innovative approaches to patient care while supporting diversity in assessment.

Since the assignment was designed in part to give students a unique way to demonstrate therapeutic principles, the prevailing hypothesis was that the infographic assignment would recognize skills different from those assessed in conventional multiple choice examinations.

\section{DESCRIPTION OF THE INNOVATION}

The 'Spotlight on Self-Care' assignment was introduced in January 2019. Conceptualization, design and implementation were accomplished by collaboration between the course coordinator and the pharmacist teaching assistant. The focus of the three-part assignment was on minor ailments and patient self-care. 
Each student was assigned one minor ailment that would typically be treated from the self-selection area of the pharmacy, such as constipation or headache. The assignment was completed by students individually. Each student created three infographics to guide pharmacists and other healthcare professionals in innovative, evidence-based and financially sustainable ways of providing medical care on that topic. The three deliverables for the assignment were: 1) a magazine insert for healthcare professionals, 2) a patient-friendly handout, and 3) a pharmacists' companion practice tool. Special emphases were placed on practicality, raising the bar on current practice standards and provision of clear approaches for integration of the students' proposals into the general community pharmacy environment.

At the beginning of the course, students were given an assignment brief that explained the rationale for the assignment, the learning objectives and the specific expectations for each of the three deliverables. The total assignment weight was $20 \%$ of the course grade. Students were allotted 8 weeks of out-of-class time to complete the work. Instructors were available by office hours and by email for questions about creation of the infographics. Out of 53 students, 4 students contacted the instructors for clarification of specific details prior to submission.

The magazine insert was targeted toward healthcare professionals and aimed to communicate an innovative approach to patient self-care in dynamic graphical form. While innovations were defined broadly as new advances in conventional self-care, over-the-counter medication use or other non-prescription therapy, the innovations were required to be evidence-based. The insert was expected to fill two $8.5^{\prime \prime} \mathrm{x}$ $11 "$ pages, or a full magazine spread.

The patient handout was intended to evaluate the students' abilities to educate patients regarding self-care with meaningful written and visual content. Patient-friendly language was defined as vocabulary at a fifth grade reading level. Instructors hypothesized that infographics would be particularly effective because images can supplement the vocabulary to increase understanding. A special focus was placed on ensuring that the handout could be used independently to assist patients in making decisions for their own self-care. As a result, the document had to be clear regarding how the information applied to special populations, such as children and geriatrics, and when to seek medical assistance from a pharmacist or physician.

Finally, the pharmacists' companion was described as a onepage practice tool aimed to streamline the patient interaction and ensure that no key questions, recommendations or messages to patients were missed. In addition, students were encouraged to provide options for comprehensive care, which could increase sales of non-prescription products for the pharmacy. For example, while a patient with a plantar wart would normally be recommended a wart remover preparation, the pharmacist should also remember to recommend the appropriate bandages and coverings to prevent the patient from picking at the wart or spreading it to others, and perhaps petroleum jelly to protect the skin around the wart.

To encourage creativity, few restrictions were placed on the formatting. Since the assignment was academic in nature, no copyrighted or trademarked materials were permitted, including brand names of medications. References to primary literature were required to support any clinical recommendations.

Very little coaching was provided to students on methods for infographic creation. In the assignment brief, students were directed to websites hosted by Piktochart (Bayan Baru, Malaysia) and Canva (Surry Hills, Australia) for training and resources. These websites provide a free and user-friendly interface for infographic creation. They also supply a number of images that can be used without copyright infringement. There were no restrictions on the software that could be used if a more flexible approach was desired.

The rubrics for evaluation of the deliverables were available to the students in the course directory (available on request). All deliverables had several consistent assessment variables, including: clinical content, logical presentation, research quality, visual elements and formatting (e.g. spelling and grammatical errors, adherence to assignment instructions). In addition, the magazine insert was assessed for innovative content, the patient handout was assessed for employing patient-friendly language and the pharmacists' companion was assessed for provision of comprehensive care.

Grading was completed by two independent evaluators for $10 \%$ of the submissions and since the results were in close agreement, the remainder of the grading was completed by one evaluator. The time investment required for grading was high. In this administration of the assignment, 53 submissions (159 infographics) were graded. On average, each assignment (totaling 3 deliverables) required 30 minutes of grading time, with brief feedback provided to students in writing.

The five highest-scoring submissions for the magazine insert deliverable were offered publication in the Pharmacy Practice and Business Magazine after peer review to showcase the innovations. Following completion, the pharmacists' companion practice tools were collated into one document covering over 20 minor ailments and distributed to students for use in their professional futures. Written consent was obtained from any students whose work was publicly shared. No ethics approval was required for analysis and reporting of deidentified student grades. 


\section{CRITICAL APPRAISAL}

Overall, 53 submissions were received. From the perspective of the instructors, the buy-in from students was strong and the assignments were all of high quality. While there were multiple students assigned to each ailment, the submissions were each uniquely focused on engaging subtopics or trends in self-care. For example, under the general topic of 'warts,' the submissions were focused on subtopics such as 'plantar warts' and 'prevention of wart transmission in children.' A minority of the assignments used a traditional, text-heavy, magazine article approach, while the majority featured dynamic workflows and bright images to draw out the messages.

By offering publication of the top five magazine inserts in the Pharmacy Practice and Business magazine, a healthy sense of competition was instilled in the classroom and students became highly engaged in the assignment. The instructors suspect that motivation for synthesizing practice innovations and completing the assignment would have been lower had this competition not been present.

With a few exceptions, students were able to adhere to the assignment instructions. Students had difficulty reporting references on infographics that were particularly busy or utilized vibrant colors. For evaluators, it was difficult to identify copyrighted materials without checking every image individually. Two students submitted images that were not created as their own work or otherwise available in the public domain. The images were referenced.

Two examples of the magazine insert are provided in Appendix 1 featuring an approach to using honey for treating cough in children and a practice guideline for implementing rapid Streptococcus antigen testing in a community pharmacy.
Across all 53 students, the mean grade was $82.3 \%$ and the standard deviation was $8.6 \%$. The grade distribution passed the test for normality. Four students requested individual appointments with the instructors to revisit and discuss their grading.

To test the hypothesis that the Spotlight assignment assessed skills that were different from those assessed in conventional examinations, each student's Spotlight grade was contrasted with that individual's average exam grade in the same course. The examinations were administered at the midpoint and at the conclusion. Both exams featured multiple choice questions and were administered using Examplify (ExamSoft, Dallas, Texas, United States). One student who had a late submission and two students who submitted copyrighted materials were excluded from the analysis since the associated grade penalties would confound the ability to answer the research question $(N=50)$. Among the remaining 50 students, the average Spotlight grade was $82.1 \%$ and the average exam grade was $81.4 \%$.

A very weak positive correlation was detected between the Spotlight grade and the corresponding exam grade for each student (Pearson linear correlation coefficient $R^{2}=0.15$, Figure $1 \mathrm{~A})$. The spaghetti plot in Figure $1 \mathrm{~B}$ highlights the variable class rankings and grades between the Spotlight assignment and the examinations. The differences in student performances across the two assessments support the hypothesis that the Spotlight assignment may be assessing skills that are different from those assessed in conventional examinations. Future investigations are required to determine the exact nature of these skills and whether they are predictive of professional success or are useful in pharmacy practice.
A

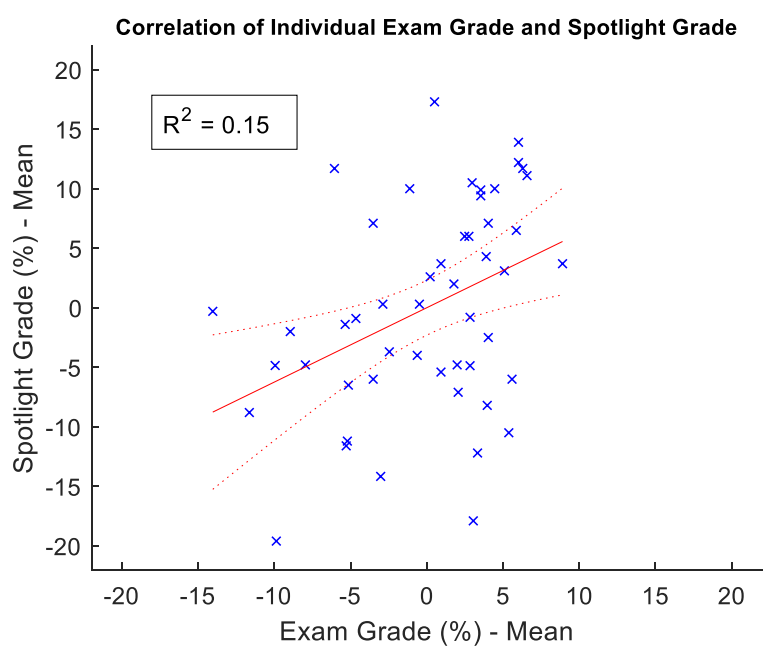

B

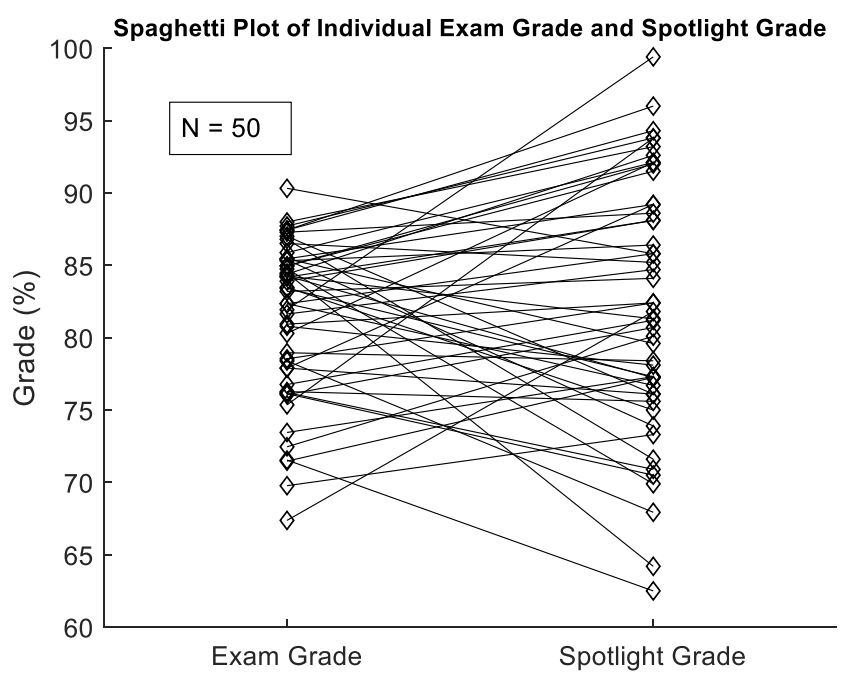

Figure 1 Statistical comparisons of Spotlight grade and average exam grade suggest that the Spotlight assignment may be assessing skills that are different from those assessed in conventional examinations. 
A subgroup analysis was also performed to evaluate whether the assignments were graded without bias toward specific ailments. No statistically significant differences in Spotlight grade were detected among the cosmetic, digestive, infectious, pain, irritant or lifestyle minor ailment subgroups (two-sample t-test).

All things considered, the key issues to address before subsequent administrations of the assignment include reducing grading time requirements and developing strategies to detect copyrighted materials. Provisionally, grading time may be reduced by eliminating the third component of the assignment (the pharmacists' companion tool), which was often a representation of the content from the magazine insert. However, this decision must be weighed against the observation that the companion tools were especially valued by students since they were designed to guide practice and were distributed to students at the conclusion of the course. Another alternative is moving the assignment from being completed individually to being completed in a small group. An anticipated challenge that did not present was the possibility that students would have a difficult time differentiating their work from the existing practice standards laid out in tertiary resources, such as the Compendium of Therapeutics for Minor Ailments. However, all of the submissions were encouragingly unique.

Future investigations into the nature of the skills gained by the students, as well as their perceptions regarding the professional value of the exercise would be important when deciding to apply this assessment in the course consistently. It is anticipated that the assignment helped students to develop communication skills that will engage audiences beyond oral and written language, namely by approaching communication dynamically with graphics. Future investigations could also examine whether performance on the assignment may be a reflection of the depth of knowledge possessed by the student, whereas multiple-choice examinations tend to select for superficial recognition memory. Summarizing vast amounts of clinical content into a succinct and powerful infographic requires strategic processing, critical thinking and thorough comprehension of the literature. ${ }^{7}$

\section{CONCLUSION}

The infographic tools put clinical content from the course into action by leveraging recent trends in effective communication. As an assessment, the infographic assignment recognized a unique profile of skills in the students that was statistically different from the profile of skills that was evaluated by the multiple-choice examinations. The diverse spectrum of assignments submitted suggests that each student took ownership over their topic.

\section{Conflicts of Interest: None.}

Funding/Support: This pilot report was funded in part by the Canadian Institutes of Health Research through the Frederick Banting and Charles Best Canada Graduate Scholarship (CGS-D).

\section{REFERENCES}

1. Marczak L, O'Rourke K, Shepard D. When and why people die in the United States, 1990-2013. JAMA. 2016;315(3):241. doi:10.1001/jama.2015.17599

2. Cox C, Kamal R, Jankiewicz A, Rousseau D. Recent trends in prescription drug costs. JAMA. 2016;315(13):1326. doi:10.1001/jama.2016.2646

3. Jones NP, Sage M, Hitchcock L. Infographics as an assignment to build digital skills in the social work classroom. J Technol Hum Serv. 2019;37(2-3):203225. doi:10.1080/15228835.2018.1552904

4. Shanks JD, Izumi B, Sun C, Martin A, Byker Shanks C. Teaching undergraduate students to visualize and communicate public health data with infographics. Front Public Health. 2017;5(315). doi:10.3389/fpubh.2017.00315

5. Falk NL. Infographic development by accelerated bachelor of science in nursing students: an innovative technology-based approach to public health education. Nurs Educ Perspect. 2016;37(5):299-301. doi:10.1097/01.nep.0000000000000066

6. Bloxham S, Boyd P. Developing effective assessment in higher education: a practical guide. Maidenhead, UK: Open University Press; 2007.

7. Van Dijk TA, Kintsch W. Strategies of discourse comprehension. New York, NY, USA: Academic Press; 1983. 


\section{Appendix 1}

\section{TIME IS HONEY \\ THE BUZZ ABOUT COUGH}

Pharmacies are often the first stop when patients develop a cough. Among the many options available for cough syrups, few certain patients, especially those looking for a natural remedy. It has been used for centuries and should not be overlooked. ${ }^{\text {? }}$,

THE FACTS

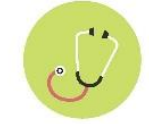

Cough is the most common reason for seeking medical

care

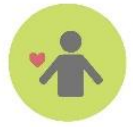

Pharmacists are primary health care providers

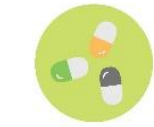

Over $70 \%$ of Canadians use Natural Health Products, with a projected 10-20\% globa

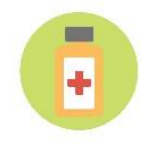

There is little to no efficacy in current over-the-counter regimens for cough

Dry vs. Productive Cough- Let it bee One of the first questions we ask our patients is to describe the nature of their cough; dry or One of the This achaic cough was critical in avoiding the development of tuberculosis. ${ }^{4}$.

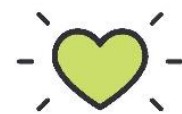

With today's modern-day medicine rendering this practice obsolete, our assessment should shift to focus on duration, and ruling-out potential red flags, including a persistent coug lasting over 3 weeks. or a cough accompanied by altered breathing such as wheezing of shorchess of breath:

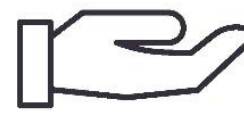

Treatment should be aimed at addressing the cough in itself, rather than overmedicating with muitt-drug cough \& cold products found over the counter. Not only are these nonprescription products cautioned for use in children, many have falled to show benefit both in regard to symptom improvement, and duration. Amongst the drug with limited evidence supporting their use, both dextromethorphan and codeine carly abuse potential, and are restricted to use in children over 6, and 12 years of age respectively. $s$ By addressing the cough symptom inividually, we can provide a more targeted and effective therapy. all the wile avoiang the potental rare. bur senous adverse events associaled with abnormal heart rates ${ }^{\circ}$

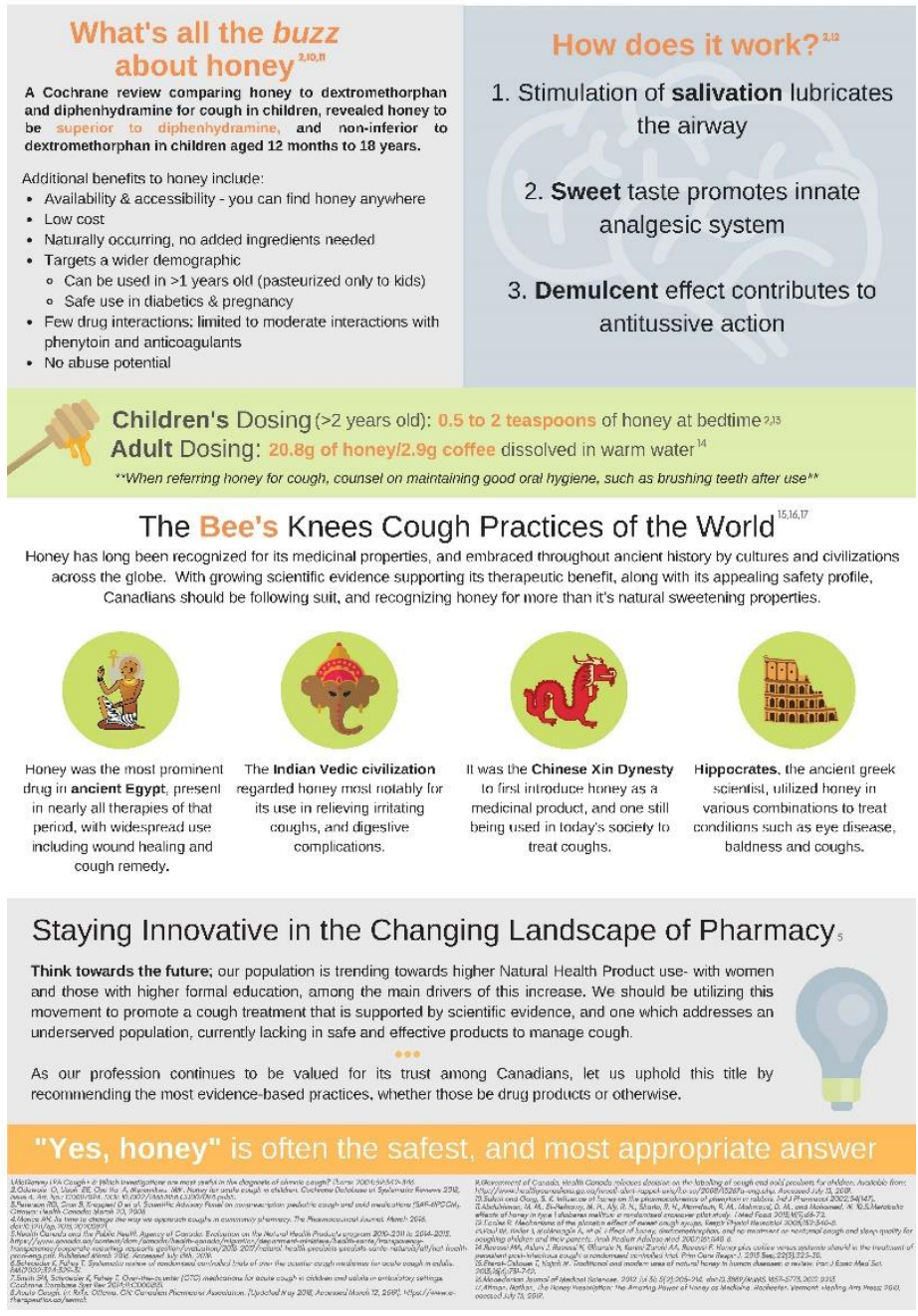




\section{PHARYNGITIS MANAGEMENT IN YOUR EOMMUNITY PHARMACY \\ By: Milena Ljumovic, PharmD Gandidate 2020}

8

Pharyngitis is an inflammation of the pharynx due to infectious or non-infectious causes.2The most occur, which is mostly caused by group A streptococcus (GAS) bacteria. ${ }^{1,2}$

GAS pharyngitis is usually self-limiting, with symptoms resolving in 3-10 days." Antibiotics can be used to.".

- Prevent rare complication

- Decrease transmission, an

rugåg.

- Shorten duration of symptoms by 1 day. RF rates are approximately 3 cases permillion a year in Canad although higher rates are seen in the First Nations population

When the etiology of pharyngitis is likely due to GAS, throat swab cultures and rapid antigen detection tests

(RADT) can be performed to confirm GAS infection an requirement for antibiotics. haryngitis, and 20-30\% in childre-

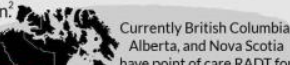
WAshe point of care RADT

- Community pharmacists are ideally positioned to help in the identification and management of patients with GAS pharyngitis. In order to prevent unnecessary antibiotic use, pharmacists should follow these 3 steps in pharyngitis management.

\section{Confirm patient has signs \& symptoms of GAS pharyngitis.}

F

GAS pharyngitis signs \& symptoms: ${ }^{146}$

Sudden and painful sore throat

Swollen s tend

58

iral pharyngitis signs \& symptoms:

- Sore throat with cough

Rhinorrhea, conjunctivitis

Hoarseness

- Oral ulcers

- Exanthema

Red Flags - refer patient to physician/hospital? ? $^{27.10}$

Fever: $>40.5^{\circ} \mathrm{C}$, persisting $>72 \mathrm{hrs}$, infant $<3$ months - "Hot potato" muffled voice, unilateral

Dysphagia, odynophagia, stridor, drooling

Shortness of breath, wheezing

. Ear pain, discharge, tugging

Barking cough
Stiff neck

sore throat

Strawberry tongue, cracked red lips,

erythema of hands and feet

Gray membrane of throat

2. Use Gentor Score to determine if GAS pharyngitis is likely.

\begin{tabular}{|c|c|}
\hline Parameter: & Points \\
\hline Cough absent & +1 \\
\hline Exudative/Swollen Tonsils & +1 \\
\hline $\begin{array}{l}\text { Nodes: swollen \& tender } \\
\quad \text { anterior cervical nodes }\end{array}$ & +1 \\
\hline Iemperature $>38^{\circ} \mathrm{C}$ & +1 \\
\hline often young $3^{*}-14$ years old & +1 \\
\hline 15 to 44 years old & 0 \\
\hline Rarely old $>45$ years old & -1 \\
\hline
\end{tabular}

Centor Score shows likelihood of GAS pharyngitis: $\leq 1=$ lower likelihood 2-3 = medium likelihood $\geq 4$ = higher likelihood

Keep in mind: Pharmacists are not trained to assess swollen lymph nodes and exudative/swollen while determining the Centor Score and making recommendations to patients. 12

NATERLOO

\section{Use Gentor score to determine need for detection tests and antihiotics.}

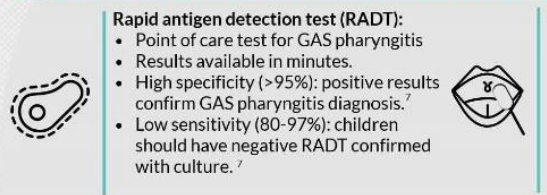

Throat swab culture:
- Gold standard: high specificity

(99\%), high sensitivity $(97 \%)^{7}$

Results available in $\geq 24$ hours.

Can identify less common

that cause pharyngitis. ${ }^{10}$

Allows for antibiotic susceptibility testing if needed.

\begin{tabular}{|c|c|c|c|}
\hline Score & Risk of GAS & \multicolumn{2}{|c|}{ Suggested Management ${ }^{3.5}$} \\
\hline$\leq 0$ & & \multirow{2}{*}{\multicolumn{2}{|c|}{$\begin{array}{l}\text { RADT/throat swab culture is not recommended. } \\
\text { Symptomatic management only. }\end{array}$}} \\
\hline 1 & $5-10 \%$ & & \\
\hline 2 & \multirow{2}{*}{$\begin{array}{l}11-17 \% \\
28-35 \%\end{array}$} & \multicolumn{2}{|c|}{ RADT/throat swab culture is recommended. } \\
\hline 3 & & \\
\hline$\geq 4$ & $51-53 \%$ & \multicolumn{2}{|c|}{$\begin{array}{l}\text { Do not start empiric antibiotics unless confirmed } \\
\text { with RADT/throat swab culture. There is still a } 50 \% \\
\text { chance that patient does not have GAS infection! }\end{array}$} \\
\hline \multicolumn{4}{|c|}{ If Centor Score 2-3 and RADT available in your pharmacy: } \\
\hline & & Positive RADT test: $^{5}$ & Negative RADT test: \\
\hline \multicolumn{2}{|c|}{$\begin{array}{c}\text { Adults } \\
\geq 15 \text { years old }\end{array}$} & \multirow{2}{*}{ tart empiric antibiotic. } & $\begin{array}{c}\text { Does not require throat } \\
\text { culture confirmation due to low } \\
\text { incidence in adults. }\end{array}$ \\
\hline \multicolumn{2}{|c|}{$\begin{array}{l}\text { Children } \\
\text { 3-14 years old }\end{array}$} & & $\begin{array}{l}\text { Confirm absence of GAS with } \\
\text { throat swab culture due to } \\
\text { higher incidence in children. }\end{array}$ \\
\hline
\end{tabular}

Keep in mind: ${ }^{12}$

in 4 individuals are carriers of GAS only be performed to confirm The diagnosis of GAS pharyngitis. diagnose GAS pharygitis. Remember that patients presenting with symptoms of viral pharyngitis or red flags should not be tested!

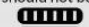

$\oplus$

Counselling tip: $:^{1.3}$ Antibiotics started within 9 days of symptom onset in confirmed $C A S$ pharyngitis will wait untill results of throat swab culture before starting antibiotics!

Nova Scotia College of Pharmacists advise pharmacists to only perform RADT, particularly in children,

$\mathbf{}\rangle \mathbf{}$ Which antibiotics are appropriate in GAS pharyngitis?

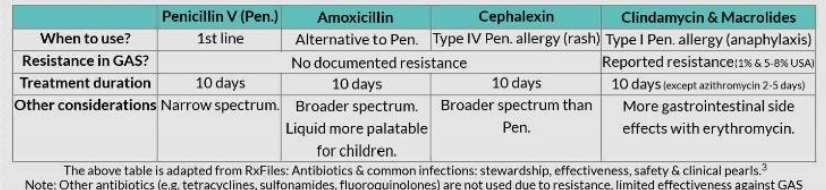

7) Symptomatic management in pharyngitis.

$$
\text { Reduce throat pain with: }
$$

$\checkmark$ Systemic analgesics (acetaminophen, ibuprofen Additionally:

$\checkmark$ Medicated lozenges (benzocaine)

$\checkmark$ Medicated sprays (phenol)

$\checkmark$ Gargling warm salt water rinses or tea

hydratio

$\checkmark$ Ensure adequate resting

$\checkmark$ Increase humidity to $>50$

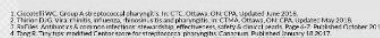

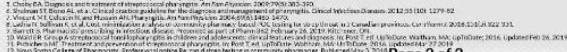

2-3 days. If symptoms

have not improved or

have worsened, refer to

physician for assessment

WATERLOO 